\title{
Quasirandom estimations of two-qubit operator-monotone-based separability probabilities
}

\author{
Paul B. Slater用 \\ Kavli Institute for Theoretical Physics, \\ University of California, \\ Santa Barbara, CA 93106-4030
}

(Dated: March 24, 2020)

\begin{abstract}
We conduct a pair of quasirandom estimations of the separability probabilities with respect to ten measures on the 15-dimensional convex set of two-qubit states, using its Euler-angle parameterization. The measures include the (non-monotone) Hilbert-Schmidt one, plus nine others based on operator monotone functions. Our results are supportive of previous assertions that the Hilbert-Schmidt and Bures (minimal monotone) separability probabilities are $\frac{8}{33} \approx 0.242424$ and $\frac{25}{341} \approx 0.0733138$, respectively, as well as suggestive of the Wigner-Yanase counterpart being $\frac{1}{20}$. However, one result appears inconsistent (much too small) with an earlier claim of ours that the separability probability associated with the operator monotone (geometric-mean) function $\sqrt{x}$ is $1-\frac{256}{27 \pi^{2}} \approx 0.0393251$. But a seeming explanation for this disparity is that the volume of states for the $\sqrt{x}$-based measure is infinite. So, the validity of the earlier conjecture-as well as an alternative one, $\frac{1}{9}\left(593-60 \pi^{2}\right) \approx 0.0915262$, we now introduce-can not be examined through the numerical approach adopted, at least perhaps not without some truncation procedure for extreme values.
\end{abstract}

PACS numbers: Valid PACS 03.67.Mn, 02.50.Cw, 02.40.Ft, 02.10.Yn, 03.65.-w

*Electronic address: slater@kitp.ucsb.edu 


\section{INTRODUCTION}

In our previous paper, "Master Lovas-Andai and equivalent formulas verifying the $\frac{8}{33}$ twoqubit Hilbert-Schmidt separability probability and companion rational-valued conjectures" [1, sec. 7.3], it was argued that the two-qubit separability probability [2] based on the measure provided by the operator monotone (geometric-mean) function $f(x)=\sqrt{x}$ would be (with the random-matrix-theoretic Dyson-index $d$ set to 2 ) given by the ratio

$$
\begin{gathered}
\mathcal{P}_{\text {sep. } \sqrt{x}}(\mathbb{C})=\frac{\int_{-1}^{1} \int_{-1}^{x} \tilde{\eta}_{d}\left(\sqrt{\frac{1-x}{1+x}} / \sqrt{\frac{1-y}{1+y}}\right)\left(1-x^{2}\right)^{-d / 4}\left(1-y^{2}\right)^{-d / 4}(x-y)^{d} \mathrm{~d} y \mathrm{~d} x}{\int_{-1}^{1} \int_{-1}^{x}\left(1-x^{2}\right)^{-d / 4}\left(1-y^{2}\right)^{-d / 4}(x-y)^{d} \mathrm{~d} y \mathrm{~d} x}= \\
\frac{\frac{\pi^{2}}{2}-\frac{128}{27}}{\frac{\pi^{2}}{2}}=1-\frac{256}{27 \pi^{2}} \approx 0.0393251 .
\end{gathered}
$$

(A twofold change-of-variables-as in [3, Thm. 2]-is employed for the integrations. At the end of this paper, we introduce an alternative hypothesis (15), (16)), as well.) The symmetric and normalized forms of operator monotone functions $f(x)$ satisfy the relation $f(x)=x f\left(\frac{1}{x}\right)$, with the associated measure (volume form) on the $n \times n$ density matrices $D$ being given by $\sqrt{\operatorname{det}\left(g_{f}(D)\right)}=\frac{1}{\sqrt{\operatorname{det}(D)}}\left(2^{\frac{1}{2}(n-1) n} \prod_{1 \leq i \leq j \leq n} c_{f}\left(\mu_{i}, \mu_{j}\right)\right)^{d / 2}$. Here, the $\mu$ 's are the $n$ eigenvalues of $D$ and $c_{f}(x, y)=\frac{1}{y f(x / y)}[3$, eq. (26)].

Equation (1) can be seen to be a modification (with $-\frac{d}{4}$ replacing $d$ as four of the six exponents) of the formula yielding the asserted (non-operator monotone [4]) Hilbert-Schmidt two-qubit separability probability (again with $d=2=2 \alpha$ ) [1, eq. (11)],

$$
\begin{gathered}
\mathcal{P}_{\text {sep. } / H S}(\mathbb{C})=\frac{\int_{-1}^{1} \int_{-1}^{x} \tilde{\chi}_{d}\left(\sqrt{\frac{1-x}{1+x}} / \sqrt{\frac{1-y}{1+y}}\right)\left(1-x^{2}\right)^{d}\left(1-y^{2}\right)^{d}(x-y)^{d} \mathrm{~d} y \mathrm{~d} x}{\int_{-1}^{1} \int_{-1}^{x}\left(1-x^{2}\right)^{d}\left(1-y^{2}\right)^{d}(x-y)^{d} \mathrm{~d} y \mathrm{~d} x}= \\
\frac{\frac{2048}{\frac{51975}{256}}}{1575}=\frac{8}{33} \approx 0.242424 .
\end{gathered}
$$

Now, Lemma 7 in [3] asserts in the two-rebit $(d=1)$ case that $\tilde{\chi}_{1}(\varepsilon)=\tilde{\eta}_{1}(\varepsilon)$ for $\varepsilon \in[0,1]$, $\varepsilon$ being the singular-value ratio [5, sec. II.A.2]. (The tilde symbol indicates normalization at $\varepsilon=1$.) Also, prior to the above pair of analyses in [1], Lovas and Andai [3] were able to formally establish for this specific $d=1$ case that these two formulas (1) and (2) yielded 
$\mathcal{P}_{\text {sep. } \sqrt{x}}(\mathbb{R}) \approx 0.26223$ and $\mathcal{P}_{\text {sep. } / H S}(\mathbb{R})=\frac{29}{64}$. For this purpose, they employed

$$
\begin{aligned}
\tilde{\chi}_{1}(\varepsilon) & =1-\frac{4}{\pi^{2}} \int_{\varepsilon}^{1}\left(s+\frac{1}{s}-\frac{1}{2}\left(s-\frac{1}{s}\right)^{2} \log \left(\frac{1+s}{1-s}\right)\right) \frac{1}{s} \mathrm{~d} s \\
& =\frac{4}{\pi^{2}} \int_{0}^{\varepsilon}\left(s+\frac{1}{s}-\frac{1}{2}\left(s-\frac{1}{s}\right)^{2} \log \left(\frac{1+s}{1-s}\right)\right) \frac{1}{s} \mathrm{~d} s .
\end{aligned}
$$

We noted in [1] that $\tilde{\chi}_{1}(\varepsilon)=\tilde{\eta}_{1}(\varepsilon)$ has a closed form,

$$
\frac{2\left(\varepsilon^{2}\left(4 \mathrm{Li}_{2}(\varepsilon)-\mathrm{Li}_{2}\left(\varepsilon^{2}\right)\right)+\varepsilon^{4}\left(-\tanh ^{-1}(\varepsilon)\right)+\varepsilon^{3}-\varepsilon+\tanh ^{-1}(\varepsilon)\right)}{\pi^{2} \varepsilon^{2}}
$$

where the polylogarithmic function is defined by the infinite sum

$$
\operatorname{Li}_{s}(z)=\sum_{k=1}^{\infty} \frac{z^{k}}{k^{s}}
$$

for arbitrary complex $s$ and for all complex arguments $z$ with $|z|<1$.

Lovas and Andai also formally established for $d=1,2$ the conjecture of Milz and Strunz [6] that the separability probability is constant for both of the indicated measures over the Bloch radii of both subsystems. Further, Slater found evidence that this constancy holds more broadly still, in the Hilbert-Schmidt case-in terms of further Casimir invariants of higher-dimensional systems [7]. In the Appendix here, we examine whether or not absolute separability probabilities might be similarly constant over the Bloch radii of the subsystems [8].

The conjecturally $(d=2)$ also equivalent "separability functions" employed in equations (1) and (2) are

$$
\tilde{\eta_{2}}(\varepsilon)=\tilde{\chi_{2}}(\varepsilon)=\frac{1}{3} \varepsilon^{2}\left(4-\varepsilon^{2}\right)
$$

More generally still, we have [1, eq. (70)]

$$
\begin{gathered}
\tilde{\chi}_{d}(\varepsilon)= \\
\frac{\varepsilon^{d} \Gamma(d+1)^{3}{ }_{3} \tilde{F}_{2}\left(-\frac{d}{2}, \frac{d}{2}, d ; \frac{d}{2}+1, \frac{3 d}{2}+1 ; \varepsilon^{2}\right)}{\Gamma\left(\frac{d}{2}+1\right)^{2}},
\end{gathered}
$$

where the regularized hypergeometric function is denoted. (Admittedly, the chain-of-reasoning leading to these functional expressions-except in the two-rebit $[d=1]$ case, due to the results of Lovas and Andai-still lacks the full rigor one would desire.) 
For the two-quater[nionic]bit instance, substituting $d=4$ into (1) and employing [1, eq. (59)]

$$
\tilde{\eta}_{4}(\varepsilon)=\frac{1}{35} \varepsilon^{4}\left(15 \varepsilon^{4}-64 \varepsilon^{2}+84\right),
$$

we reported [1, eq. (88)] the ratio of $\frac{4 \pi^{2}}{3}-\frac{5513}{420}$ to $1.478504859 \times 10^{13}$, yielding (the "infinitesimal") result

$$
\mathcal{P}_{P P T . \sqrt{x}}(\mathbb{Q})=2.2510618339 \times 10^{-15} .
$$

However, it now appears to us that the denominator is fallacious, and simply evaluates to $\infty$.

For still further extensions of these separability functions from Hilbert-Schmidt to more general induced measures, see [5]. By way of example, for the $d=2$ two-qubit setting with the induced measure parameter $k=1$ (where $k=0$ corresponds to Hilbert-Schmidt measure), we have an extended formula $\tilde{\chi}_{2,1}(\varepsilon)=\frac{1}{4} \varepsilon^{2}\left(3-\varepsilon^{2}\right)^{2}$, yielding a separability probability of $\frac{61}{143}=\frac{61}{11 \cdot 13} \approx 0.426573$.

\section{ANALYSES}

We now report a pair of numerical analyses in which we estimate the two-qubit (that is, $d=2$ ) separability probabilities associated with the Hilbert-Schmidt measure and nine operator monotone functions [9, 10], among them the $\sqrt{x}$ one already noted, as well as the Bures, Kubo-Mori and Wigner-Yanase [11] ones of strong interest. (Andai has a list from which we drew [9, sec. 4], and the order of which we largely follow.)

Though the pair of analyses conducted is certainly strongly supportive of our previous assertions that the two-qubit Hilbert-Schmidt and Bures separability probabilities are $\frac{8}{33}$ [12] and $\frac{25}{341}$ [13], respectively, they do strongly differ (in being much smaller) from the

$\mathcal{P}_{\text {sep. } \sqrt{x}}(\mathbb{C})=1-\frac{256}{27 \pi^{2}} \approx 0.0393251$ claim. However, upon further reflection, we suspect that this may be an artifact of the infinite-volume property [9] of the $\sqrt{x}$ measure, which needs to be addressed in a more nuanced numerical manner, if at all possible.

It is of interest to compare and contrast the subject matter and methodologies of the present study with that of two of our papers from 2005, "Silver mean conjectures for 15-d volumes and 14-d hyperareas of the separable two-qubit systems" [14] and "Qubit-qutrit separability probability ratios" [15]. These studies employed a different (Tezuka-Faure) approach to quasi-Monte Carlo estimation [16] than the quasirandom one here, while obtaining volume and hyperarea estimates for various operator monotone-based measures. However, in neither 
study was the geometric-mean-based measure $f(x)=\sqrt{x}$-of central concern here-examined. Also, issues of absolute separability probabilities were not studied as they had been in our later 2009 paper, "Eigenvalues, Separability and Absolute Separability of Two-Qubit States" [17], and in the Appendix below.

To conduct the pair of estimations of ten separability probabilities, we employed the SU(4)based Euler-angle parameterization [18] of the 15-dimensional convex set of two-qubit density matrices. Though in the past, we have, in fact, extensively employed this parameterization in separability probability analyses [8, 19, 20], we have more recently [13, 21, 22, relied upon the Ginibre-ensemble approach of Osipov, Sommers and Życzkowski for generating random states [23]. However, their procedure is designed for Hilbert-Schmidt and Bures measures and not apparently for the other operator monotone measures to be investigated here. (Ginibre ensembles can also be employed for the generation of random density matrices with respect to the extension $[k \neq 0]$ of Hilbert-Schmidt to induced measures [24].)

In particular, since we wanted to numerically investigate our conjecture (2) as to the value of $\mathcal{P}_{\text {sep. } \sqrt{x}}(\mathbb{C})$, it seemed appropriate to revert to the use of the Euler-angle parameterization. Let us further note that in the two-qubit setting, rather than 15 (uniformly-distributed) random numbers (needed for 12 Euler angles $\left[\alpha_{i}, i=1, \ldots, 12\right]$ and 3 eigenvalues $\left[\lambda_{i}\right.$, $i=1,2,3$, with $\left.\left.\lambda_{4}=1-\lambda_{1}-\lambda_{2}-\lambda_{3}\right]\right)$ at each iteration, in the Ginibre-ensemble approach, the considerably larger numbers of 32 and 64 (normally-distributed) ones are required in the Hilbert-Schmidt and Bures cases, respectively. On the other hand, in the Euler-angle setting, each realization needs to be weighted by the product of the Haar [18, eq. (34)]

$$
\sin \left(2 \alpha_{2}\right) \sin \left(\alpha_{4}\right) \sin ^{5}\left(\alpha_{6}\right) \sin \left(2 \alpha_{8}\right) \sin ^{3}\left(\alpha_{10}\right) \cos ^{3}\left(\alpha_{4}\right) \cos \left(\alpha_{6}\right) \cos \left(\alpha_{10}\right)
$$

and eigenvalue measures,

$$
\frac{\lambda_{1}^{3}\left(\lambda_{1}-\lambda_{2}\right)^{2} \lambda_{2}^{2}\left(\lambda_{1}-\lambda_{3}\right)^{2}\left(\lambda_{2}-\lambda_{3}\right)^{2} \lambda_{3}\left(\lambda_{1}-\lambda_{4}\right)^{2}\left(\lambda_{2}-\lambda_{4}\right)^{2}\left(\lambda_{3}-\lambda_{4}\right)^{2}}{\left(\lambda_{1} \lambda_{2} \lambda_{3} \lambda_{4}\right)^{7 / 2} f\left(\frac{\lambda_{1}}{\lambda_{2}}\right) f\left(\frac{\lambda_{1}}{\lambda_{3}}\right) f\left(\frac{\lambda_{2}}{\lambda_{3}}\right) f\left(\frac{\lambda_{1}}{\lambda_{4}}\right) f\left(\frac{\lambda_{2}}{\lambda_{4}}\right) f\left(\frac{\lambda_{3}}{\lambda_{4}}\right)},
$$

while in the Ginibre-ensemble alternative, each $4 \times 4$ density matrix produced simply receives equal weight. It would clearly be of interest to evaluate the relative merits of the two methodologies in their common domains of application.

Further, we used the quasirandom (generalized golden-ratio) estimation methodology recently developed by Martin Roberts [13, 25, 26] with its single free $\alpha_{0} \in[0,1]$ parameter set to $\frac{1}{4}$ in one analysis and $\frac{3}{4}$ in the companion one. At each iteration of these two procedures, 
we obtain 15 numbers in $[0,1]$. Interestingly, we were able to jointly use (multiplying by $\pi$ or $\frac{\pi}{2}$, as appropriate) 12 of them for the Euler-angle parameters, and the other 3 (by sorting them, appending 0 and 1, and taking differences) to obtain the four eigenvalues constrained to sum to 1. (To greatly speed our computations, we employed the Compile[, CompilationTarget $\longrightarrow$ "C", RuntimeAttributes $\longrightarrow$ Listable, Parallelization $\longrightarrow$ True] feature of Mathematica, but doing so restricted us to the use of single/normal precision. As the estimation proceeds, and greater successive are employed as seeds, the occurrence of overflows in the computations noticeably increases. These limited instances have to be discarded, but presumably no systematic effects are introduced by doing so.)

\section{A. Quasirandom procedure}

As noted, we have employed an "open-ended" sequence (based on extensions of the golden ratio [27]) recently introduced by Martin Roberts in the detailed presentation "The Unreasonable Effectiveness of Quasirandom Sequences" [25].

Roberts notes: "The solution to the $d$-dimensional problem, depends on a special constant $\phi_{d}$, where $\phi_{d}$ is the value of the smallest, positive real-value of x such that"

$$
x^{d+1}=x+1,
$$

( $d=1$, yielding the golden ratio, and $d=2$, the "plastic constant" [26]). The $n$-th terms in the quasirandom (Korobov) sequence take the form

$$
\left(\alpha_{0}+n \alpha\right) \bmod 1, n=1,2,3, \ldots
$$

where we have the $d$-dimensional vector,

$$
\alpha=\left(\frac{1}{\phi_{d}}, \frac{1}{\phi_{d}^{2}}, \frac{1}{\phi_{d}^{3}}, \ldots, \frac{1}{\phi_{d}^{d}}\right) . "
$$

The additive constant $\alpha_{0}$ is typically taken to be 0 . "However, there are some arguments, relating to symmetry, that suggest that $\alpha_{0}=\frac{1}{2}$ is a better choice," Roberts observes.

In [13], such points uniformly distributed in the $d$-dimensional hypercube $[0,1]^{d}$, were converted, using an algoirthm of Henrik Schumacher [28] to (quasirandomly distributed) normal variates, required for the generation of Ginibre ensembles. However, here, since we rely upon the Euler-angle, such a conversion is not required. 


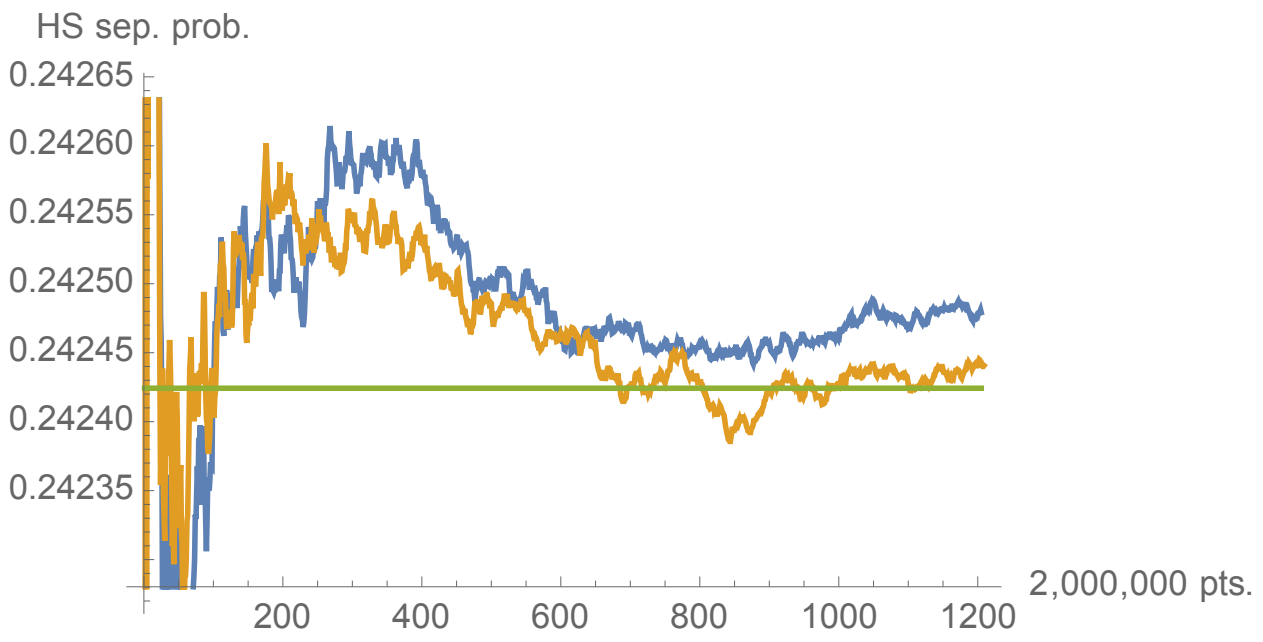

FIG. 1: Pair of estimates with respect to the Hilbert-Schmidt measure along with the conjectured value of $\frac{8}{33}$. The $x$ axis here-and in all our figures but the last two are labeled in units of two million points, so the label 1200 corresponds to two billion four hundred million points generated.

\section{B. Results}

In Fig. 1 we show the pair of quasirandom estimates obtained with respect to the HilbertSchmidt measure along with the conjectured value of $\frac{8}{33}[12$. The $x$ axis here-and in all our figures but the last two are labeled in units of two million points, so the label 1200 corresponds to two billion four hundred million points generated. We conducted paired analyses, since it was computationally convenient given the two Mathematica kernels available to us. The blue (largely greater-valued) curve is based on the Roberts parameter $\alpha_{0}=\frac{1}{4}$, and the other (orange) based on $\alpha_{0}=\frac{3}{4}$. The (arithmetic) average of the last two values is 0.24246 .

In Fig. 2 we show the pair of estimates with respect to the Bures (minimal monotone) $\left(f(x)=\frac{x+1}{2}\right)$ measure accompanied by the conjectured value of $\frac{25}{341}$ [13].

Further, in Fig. 3 we show the pair of (near-zero) estimates with respect to the maximal $\left(f(x)=\frac{2 x}{x+1}\right)$ measure. The volume of two-qubit states associated with this measure is, however, apparently infinite [3, sec. 4].

In Fig. 4 we show the pair of estimates with respect to the Kubo-Mori $\left(f(x)=\frac{x-1}{\log (x)}\right)$ measure, while, in Fig. 5 we show the pair of estimates obtained using the geometric mean $(f(x)=\sqrt{x})$ measure.

This last plot would appear to constitute evidence against the validity of the conjecture 


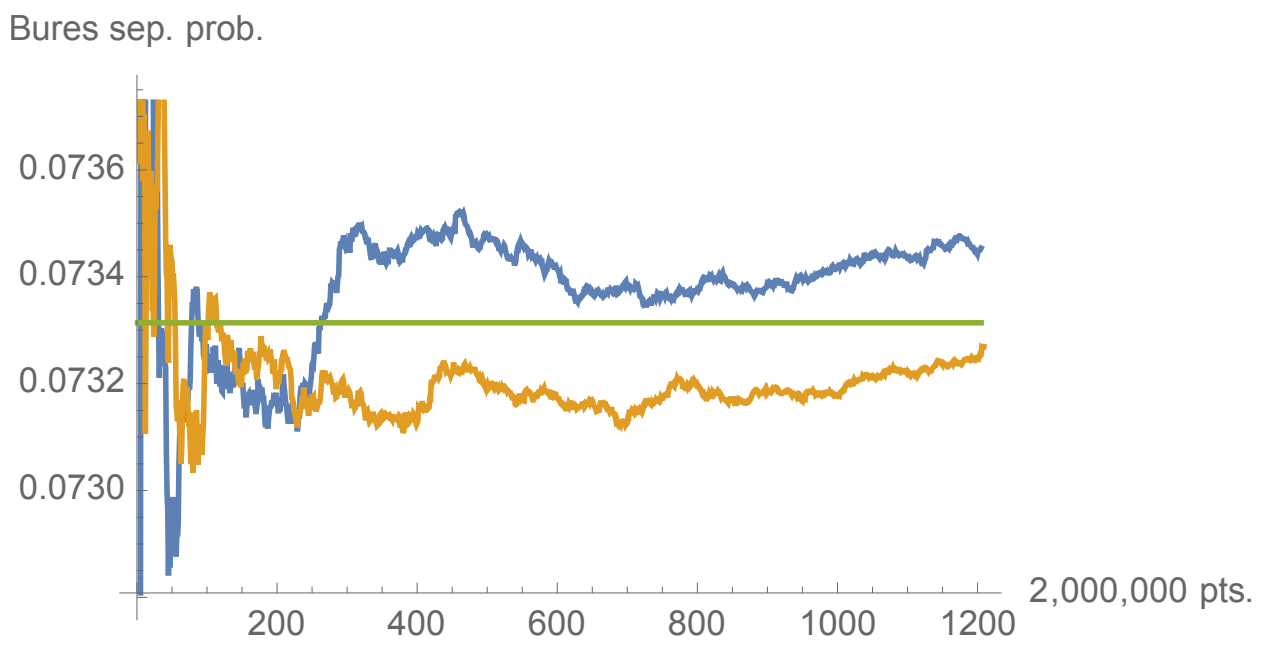

FIG. 2: Pair of estimates with respect to the Bures $\left(f(x)=\frac{x+1}{2}\right)$ measure along with the conjectured value of $\frac{25}{341}$

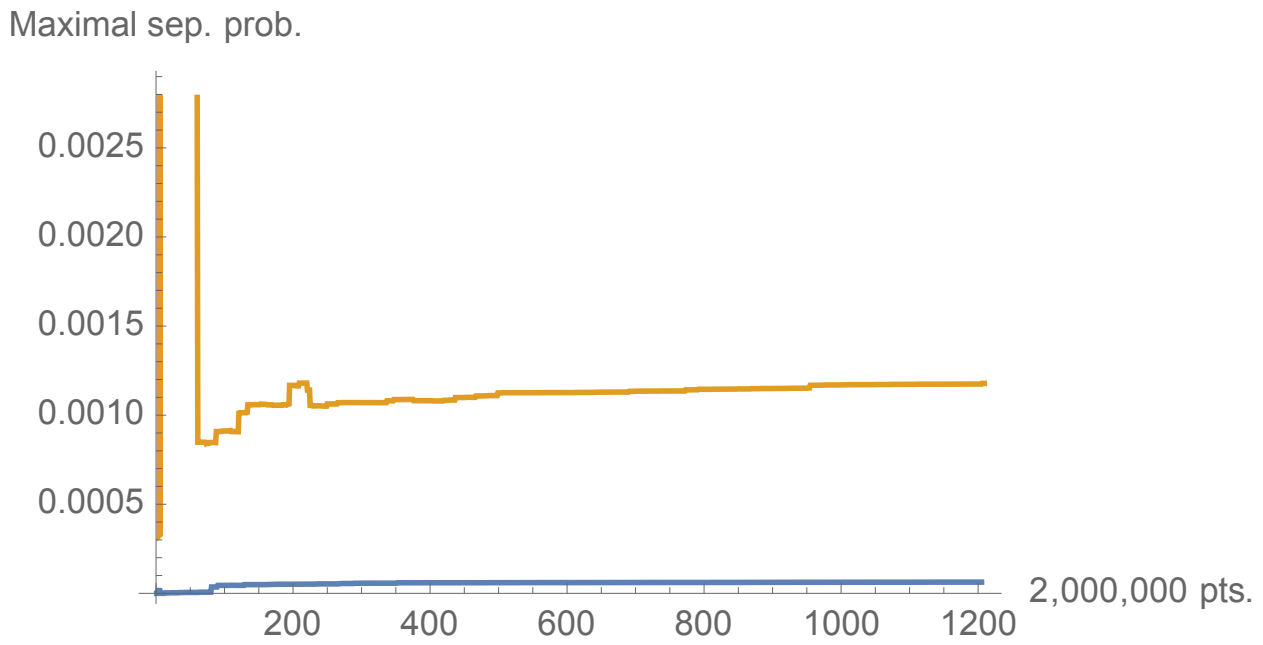

FIG. 3: Pair of estimates with respect to the maximal $\left(f(x)=\frac{2 x}{x+1}\right)$ measure

that $\mathcal{P}_{\text {sep. } \sqrt{x}}(\mathbb{C})=1-\frac{256}{27 \pi^{2}} \approx 0.0393251$ given in eq. (2). However, we must note that a seeming explanation for this inconsistency is that the volume of states for the $\sqrt{x}$-based measure is infinite, as observed by Lovas and Andai [3, sec. 5]. Perhaps, a numerical analysis in which a threshold on the magnitude of the $\sqrt{x}$ measure sampled is imposed would be appropriate. Another strategy might be to require that no randomly generated eigenvalue employed be less than a certain magnitude. Further, the quite small estimated separability probability $(\approx 0.005)$ in Fig. 5 is rather surprising, since in the two-rebit $(d=1)$ scenario $\mathcal{P}_{\text {sep. } \sqrt{x}}(\mathbb{R}) \approx 0.26223$ and $\mathcal{P}_{\text {sep } / H S}(\mathbb{R})=\frac{29}{64} \approx 0.453125$ are of similar magnitudes. 
Kubo-Mori sep. prob.

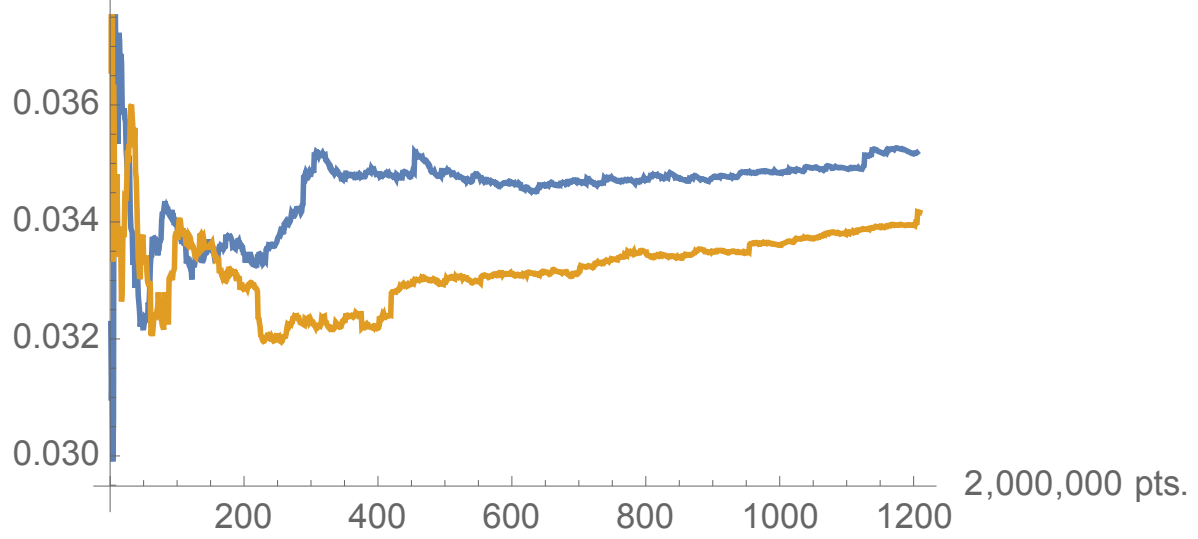

FIG. 4: Pair of estimates with respect to the Kubo-Mori $\left(f(x)=\frac{x-1}{\log (x)}\right)$ measure Geom. sep. prob.

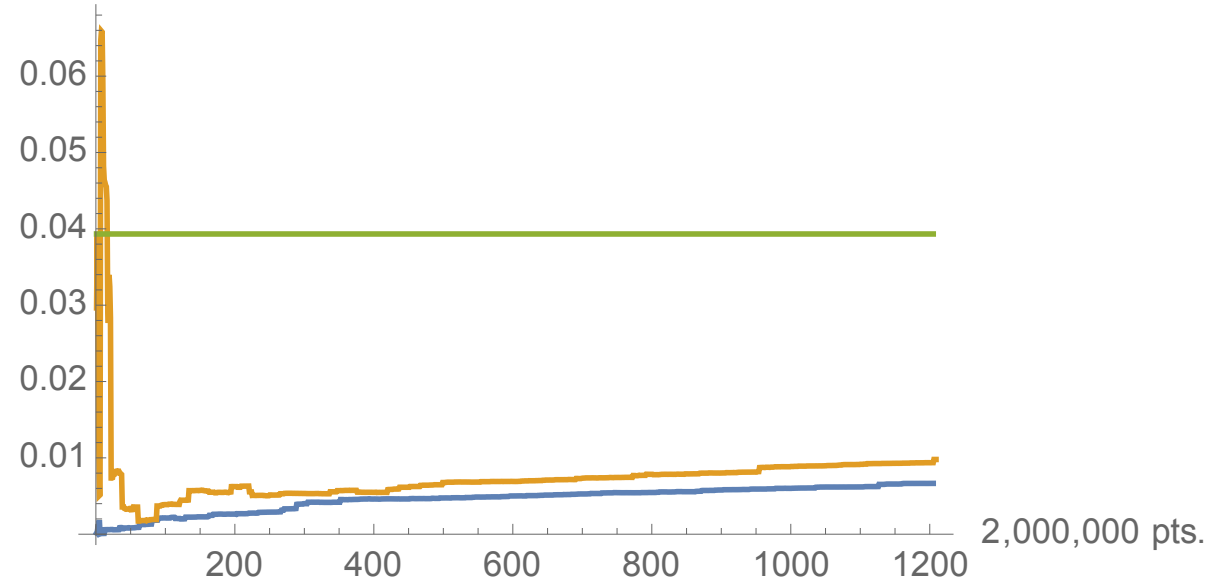

FIG. 5: Pair of estimates with respect to the geometric mean $(\sqrt{x}$-based $)$ measure along with the conjectured value of $1-\frac{256}{27 \pi^{2}} \approx 0.0393251$-given in 2

Relatedly, Lovas and Andai stated-with regard to the $\sqrt{x}$-measure-that "We show that the volumes of rebit-rebit and qubit-qubit states are infinite, although there is a simple and reasonable method to define the separability probabilities. We present integral formulas for separability probabilities in this setting, too." Also, they wrote: "Contrary to the $2 \times 2$ case ... the volume of the statistical manifold $\left(\mathcal{D}_{4, K}, g_{\sqrt{x}}\right)$ is infinite in both of the real and complex cases because $\eta_{d}(1)=\infty$ and the volume admits the following factorization

$$
\operatorname{Vol}_{\sqrt{x}}\left(\mathcal{D}_{4, \mathbb{K}}\right) .=4 \eta_{d}(1) \times \int_{\mathcal{D}_{2, \mathbb{K}}} \operatorname{det}(D)^{\frac{5}{2} d-\frac{d^{2}}{2}-1} \mathrm{~d} \lambda_{d+1}(D) \times \int_{\varepsilon_{2, \mathbb{K}}} \operatorname{det}\left(I-Y^{2}\right)^{\frac{d-2}{4}} \mathrm{~d} \lambda_{d+2}(Y)^{\prime}
$$




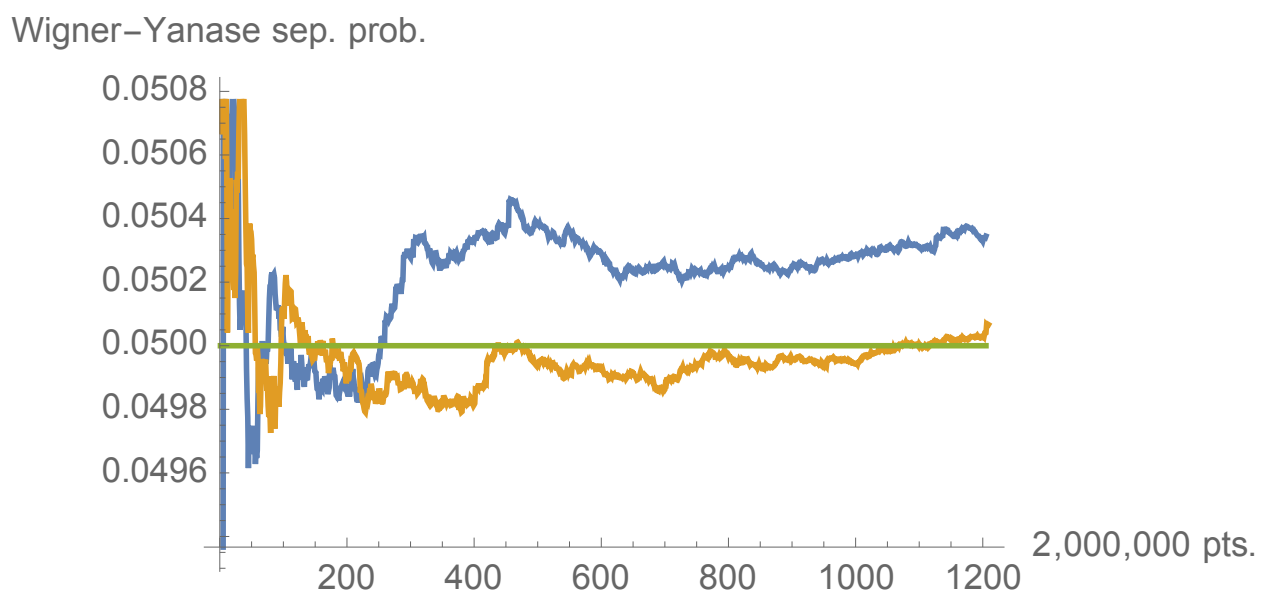

FIG. 6: Pair of estimates with respect to the Wigner-Yanase $f(x)=\left(\frac{1}{4}(\sqrt{x}+1)^{2}\right)$ measure, along with well-fitting $\frac{1}{20}$ line

(For further reference, with regard to the alternative hypothesis given in $([15),(16))$ below, note the presence of the exponents $\frac{5}{2} d-\frac{d^{2}}{2}-1$ and $\frac{d-2}{4}$, equalling 2 and 0 , respectively, for $d=2$.

In Fig. 6 we show the pair of estimates (interestingly close to $\frac{1}{20}$ ) with respect to the Wigner-Yanase $\left(f(x)=\frac{1}{4}(\sqrt{x}+1)^{2}\right)$ measure. (A third estimation-now with Roberts parameter $\alpha_{0}=0$ and 316 million realizations-also gave us a close estimate of 0.0499207 . Additionally, a fourth [Tezuka-Faure sequence quasi-Monte Carlo] estimate of 0.0503391 was reported in Table II of our 2005 study [14]. In that table, estimates of 0.0346801 and 0.0609965 were reported for the Kubo-Mori and identric measures.)

In Fig. 7 we present the pair of estimates with respect to the $f(x)=\frac{2(x-1) \sqrt{x}}{(x+1) \log (x)}$ measure. Again, the volume of two-qubit states associated with this measure is apparently infinite [3, sec. 4].

In Fig. 8 we show the pair of estimates with respect to the $f(x)=\frac{x^{2}+6 x+1}{4 x+4}$ measure, along with the closely-fitted value of $\frac{1}{21}$. (This function is the arithmetic average of the ones for the minimal (Bures) $-\frac{x+1}{2}$-and maximal- $-\frac{2 x}{x+1}-$ measures, as noted in [14, eq. (14)].)

In Fig. 9 we show the pair of estimates with respect to the Morozova-Chentsov $(f(x)=$ $\left.\frac{2(x-1)^{2}}{(x+1) \log ^{2}(x)}\right)$ measure [29, sec. II.B].

Then, in Fig. 10 we display the pair of estimates with respect to the "Grosse-KrattenthalerSlater" (GKS/quasi-Bures) $\left(f(x)=\frac{x^{\frac{x}{x-1}}}{e}\right)$ measure-also more broadly termed the "identric" measure. $\left(\frac{2}{33} \approx 0.0606061\right.$ is a closely-fitting value to the estimates). This mean appears 


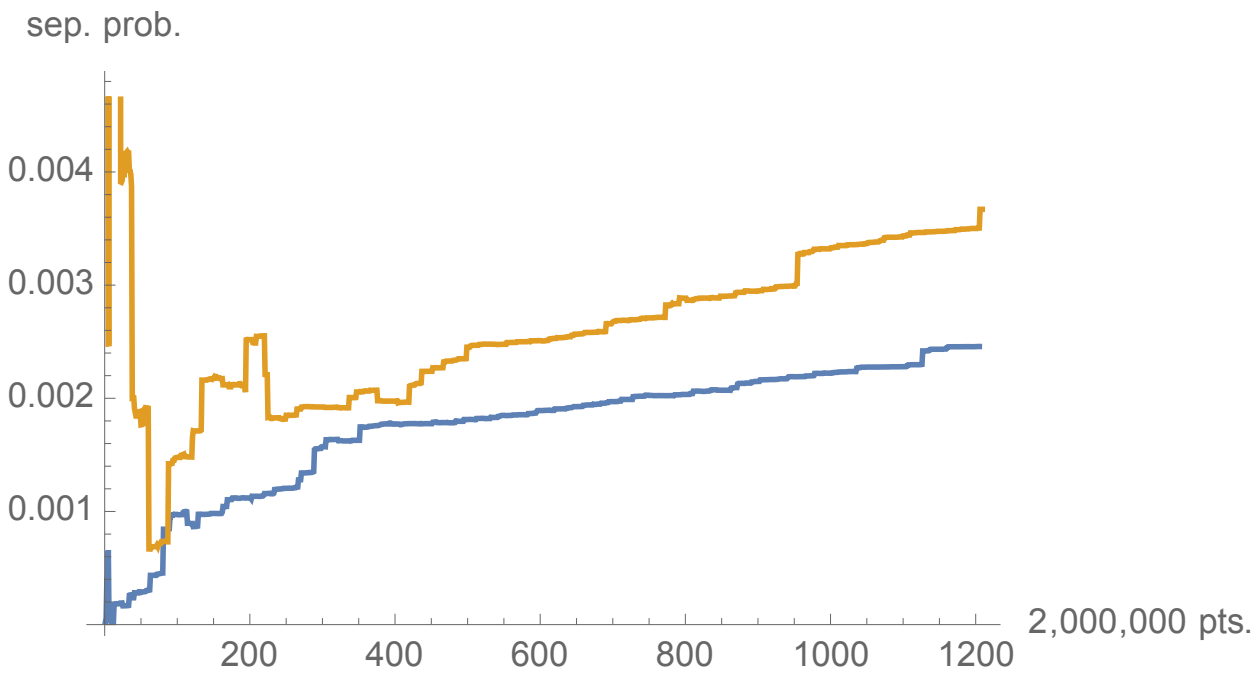

FIG. 7: Pair of estimates with respect to the $f(x)=\frac{2(x-1) \sqrt{x}}{(x+1) \log (x)}$ measure

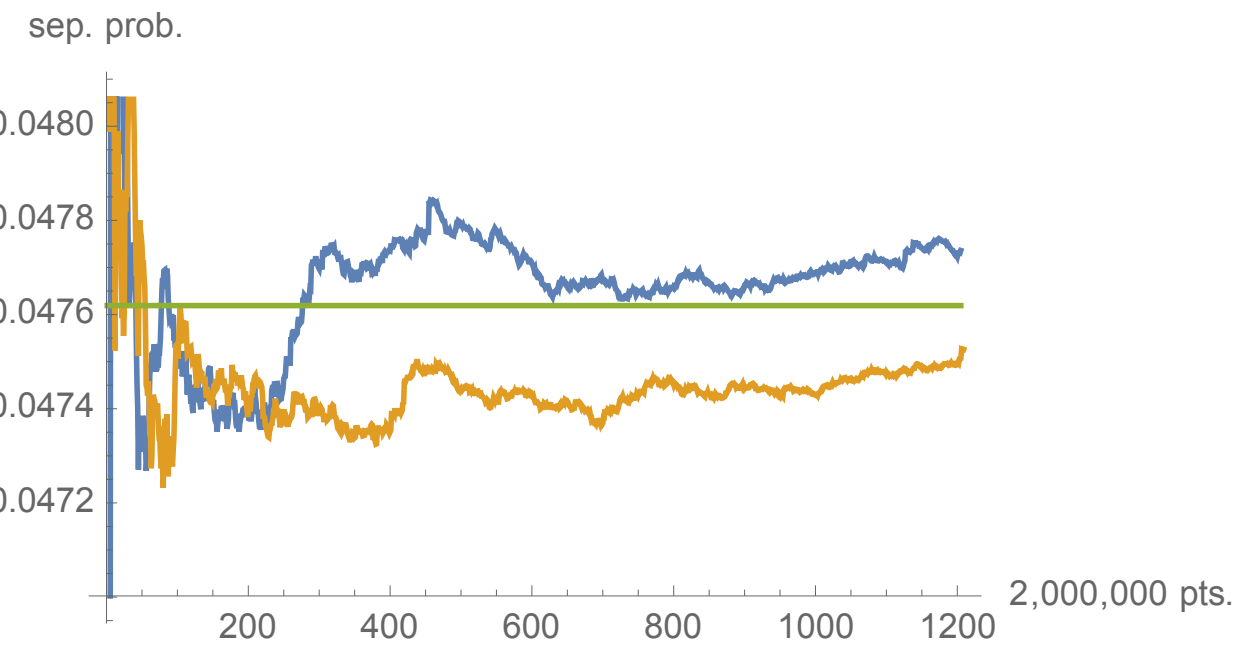

FIG. 8: Pair of estimates with respect to the $f(x)=\frac{x^{2}+6 x+1}{4 x+4}$ measure, along with the closely-fitted value of $\frac{1}{21}$

to play an important role in universal quantum coding [30, sec. IV.B] [15], in yielding the common asymptotic minimax and maximin redundancy.

So, at this point in time, we have strongly compelling-yet no formal proof-that the Hilbert-Schmidt two-qubit separability probability is $\frac{8}{33}$ [1, 12], and interesting numerical evidence pointing to the Bures counterpart being $\frac{25}{341}$ [13]. Further, the Wigner-Yanase probability appears to be quite close to $\frac{1}{20}=0.05$. Also, we indicate that $\frac{1}{21}$ and $\frac{2}{33}$ provide close-fitting values in the arithmetic and identric cases. 
Morozova-Chentsov sep. prob.

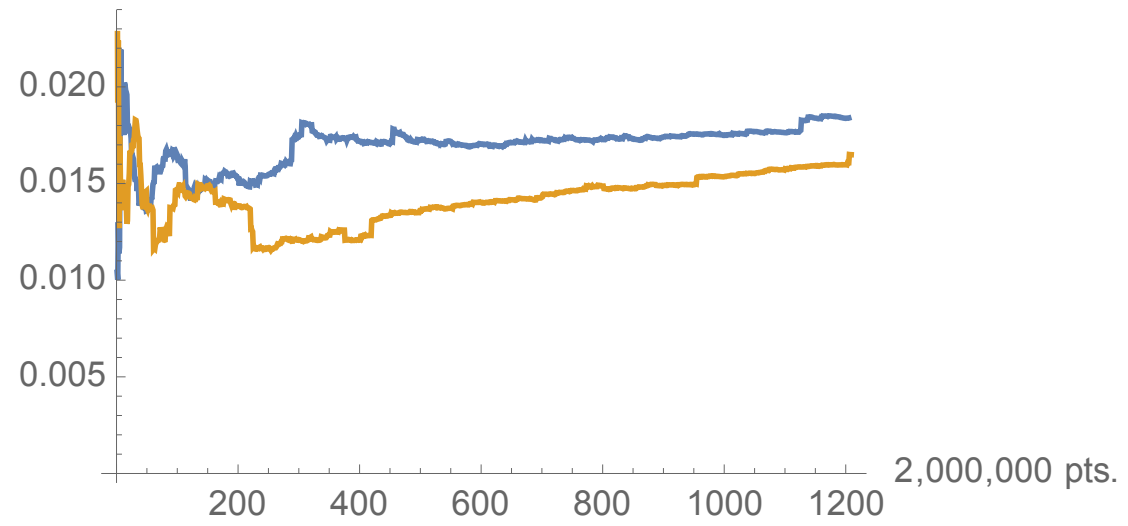

FIG. 9: Pair of estimates with respect to the Morozova-Chentsov $\left(f(x)=\frac{2(x-1)^{2}}{(x+1) \log ^{2}(x)}\right)$ measure [29]

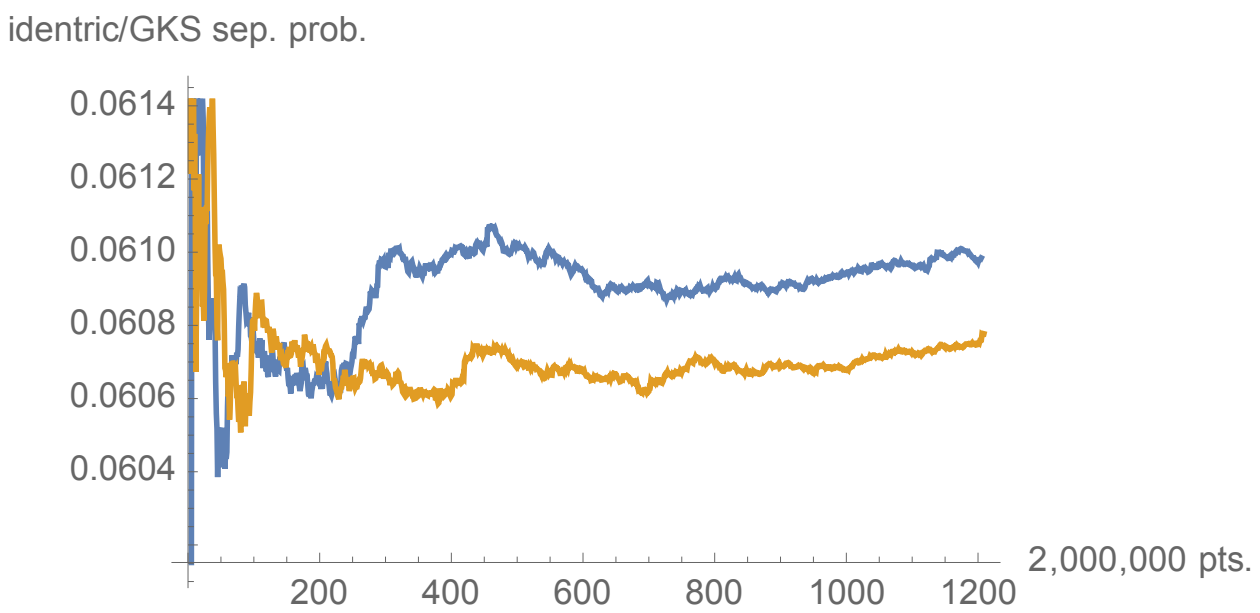

FIG. 10: Pair of estimates with respect to the Grosse-Krattenthaler-Slater (GKS)/quasi-Bures $\left(f(x)=\frac{x^{\frac{x}{x-1}}}{e}\right)$ measure

If we standardize our estimate of the Bures total (separable plus entangled) volume of two-qubit states to equal 1, then the accompanying estimate of the Kubo-Mori volume is 60.7832 as large, of the Wigner-Yanase volume 7.69711 as large, and the identric/GKS volume, 2.87957 as large. In the single-qubit case, Andai gives the Bures, Kubo-Mori, Wigner-Yanase and Morozova-Chentsov volumes as $\pi^{2}, 2 \pi^{2}, 4 \pi(\pi-2)$ and $\frac{\pi^{4}}{2}$, respectively. Based on the list of single-qubit volumes following Corollary 1 in [9], we would anticipate that the maximal, geometric and $\frac{2(x-1) \sqrt{x}}{(x+1) \log (x)}$-based volumes are all infinite. Along such lines, the estimates of how much larger they are than the Bures that we obtained were $5.38871 \times 10^{18}, 2.80034 \times 10^{7}$ and $4.65758 \times 10^{10}$, respectively. 
Upon re-examination of the detailed argument of Lovas and Andai [3], in particular their Corollary 3 , we considered the possibility that rather than the geometric-mean $(\sqrt{x}$-based) two-qubit conjecture (1), we might have (again with the random-matrix Dyson-index $d$ set to 2) the formula (replacing the four occurrences in (1) of $-d / 4$ with $\frac{d-2}{4}$ )

$$
\begin{gathered}
\mathcal{P}_{\text {sep. } \sqrt{x}}(\mathbb{C})=\frac{\int_{-1}^{1} \int_{-1}^{x} \tilde{\eta}_{d}\left(\sqrt{\frac{1-x}{1+x}} / \sqrt{\frac{1-y}{1+y}}\right)\left(1-x^{2}\right)^{\frac{d-2}{4}}\left(1-y^{2}\right)^{\frac{d-2}{4}}(x-y)^{d} \mathrm{~d} y \mathrm{~d} x}{\int_{-1}^{1} \int_{-1}^{x}\left(1-x^{2}\right)^{\frac{d-2}{4}}\left(1-y^{2}\right)^{\frac{d-2}{4}}(x-y)^{d} \mathrm{~d} y \mathrm{~d} x}= \\
\frac{-\frac{4}{27}\left(60 \pi^{2}-593\right)}{\frac{4}{3}}=\frac{1}{9}\left(593-60 \pi^{2}\right) \approx 0.0915262 .
\end{gathered}
$$

(We note that 593 is prime.) For the two-rebit $[d=1]$ case, the two formulas are simply equivalent-that is, $-d / 4=\frac{d-2}{4}=-\frac{1}{4}$. Also, both these conjectures assume that the formally proven result $\tilde{\chi}_{1}(\varepsilon)=\tilde{\eta}_{1}(\varepsilon)$ [3, Lemma 7, App. B] can be extended to the proposition that $\tilde{\chi}_{2}(\varepsilon)=\tilde{\eta}_{2}(\varepsilon)$. For $d=2$, the terms $\left(1-x^{2}\right)$ and $\left(1-y^{2}\right)$ simply "disappear" from the integrands in (15)-an apparent further manifestation of simplification in the standard 15-dimensional convex set of two-qubits framework.

A separability probability as large as 0.0915262 did seem somewhat somewhat surprising to us, as we had come to believe that the Bures (minimal monotone) two-qubit one-conjectured to be $\frac{25}{341} \approx 0.0733138$-is the largest among the family of operator monotone measures. Continuing with this $\frac{d-2}{4}$-ansatz, the two-quaterbit separability probability-using (8)-would then be the ratio of $\frac{3342341 \pi^{2}}{64}-\frac{1136525312}{2205}$ to $\frac{5 \pi^{2}}{64}$, that is, $\frac{3342341}{5}-\frac{72737619968}{11025 \pi^{2}} \approx 0.014015$. (We have $72737619968=2^{23} \cdot 13 \cdot 23 \cdot 29$ and $11025=3^{2} \cdot 5^{2} \cdot 7^{2}=105^{2}$, while 3342341 is itself prime.)

It would certainly be a lofty goal to seek a higher-order function ("functional") $f$ that given any operator monotone function would return the corresponding two-qubit separability probability. In regard to such a line of thought, J. E. Pascoe wrote: "It might be useful to consider the fact that operator monotone functions are exactly self maps of the upper half plane, and therefore have nice integral representations. In Peter Lax 'Functional Analysis' book, I think these are called 'Nevanlinna representations'. To make a long story short, this would make your function $f$ depend on a real number $a$, a nonnegative $b$ and a positive measure on the real line $\mu . "$ 


\section{Appendix A: Absolute separability probabilities}

In [17, eq. (34)], making use of the eigenvalue inequality formula [31, eq. (3)],

$$
\lambda_{1} \leq \lambda_{3}+2 \sqrt{\lambda_{2} \lambda_{4}}
$$

we reported a formula for the Hilbert-Schmidt two-qubit absolute separability probability [32, 33]-measuring the proportion of states that can not be entangled by unitary transformationsof the 15-dimensional convex set of two-qubit states. It was later further condensed to

$$
\begin{gathered}
\frac{29902415923}{497664}+\frac{-3217542976+5120883075 \pi-16386825840 \tan ^{-1}(\sqrt{2})}{32768 \sqrt{2}}=\quad \text { (A2) } \\
\frac{32(29902415923-24433216974 \sqrt{2})+248874917445 \sqrt{2}\left(5 \pi-16 \tan ^{-1}(\sqrt{2})\right)}{2^{16} \cdot 3^{5}} \approx 0.00365826,
\end{gathered}
$$

much smaller than the combined (absolute and non-absolute) separability probability of $\frac{8}{33} \approx 0.242424$. ("[C]opious use was made of trigonometric identities involving the tetrahedral dihedral angle $\phi=\cos ^{-1}\left(\frac{1}{3}\right)$ ", assisted by V. Jovovic. Equation A2 here corrects a misprint in eq. (A2) in [1]. We also confirmed this highly challenging-to-obtain 2009 result, at least to high numerical precision, in a de novo analysis.)

In [17, sec. III.C], we also gave a Bures two-qubit absolute separability probability estimate of 0.000161792. (Startingly, in essentially total agreement with these last two results, in [34, Table 2], Khvedelidze and Rogojin reported Hilbert-Schmidt and Bures estimates of 0.00365826 and 0.000161792 , respectively.)

In certain of our 15-dimensional quasirandom estimations conducted earlier here, we also collaterally estimated the lower (4)-dimensional absolute separability probabilities (rather than in a de novo 4-D analysis). For instance, in Fig. 11, we now show our quasirandom estimation (with $\alpha_{0}=0$ ) of the Hilbert-Schmidt two-qubit absolute separability probability along with the predicted value $\mathrm{A} 2$.

In Fig. 12, we show the deviations about the-as indicated-previously tabulated value of 0.000161792 of a quasirandom estimation (with $\alpha_{0}=0$ ) of the Bures two-qubit absolute separability probability.

In Fig. 13, we show a quasirandom estimation (again with $\alpha_{0}=0$ ) of the Kubo-Mori twoqubit absolute separability probability and in Fig. 14, we present a quasirandom estimation (with $\alpha_{0}=0$ ) of the GKS/identric two-qubit absolute separability probability. 
HS abs. sep. prob.

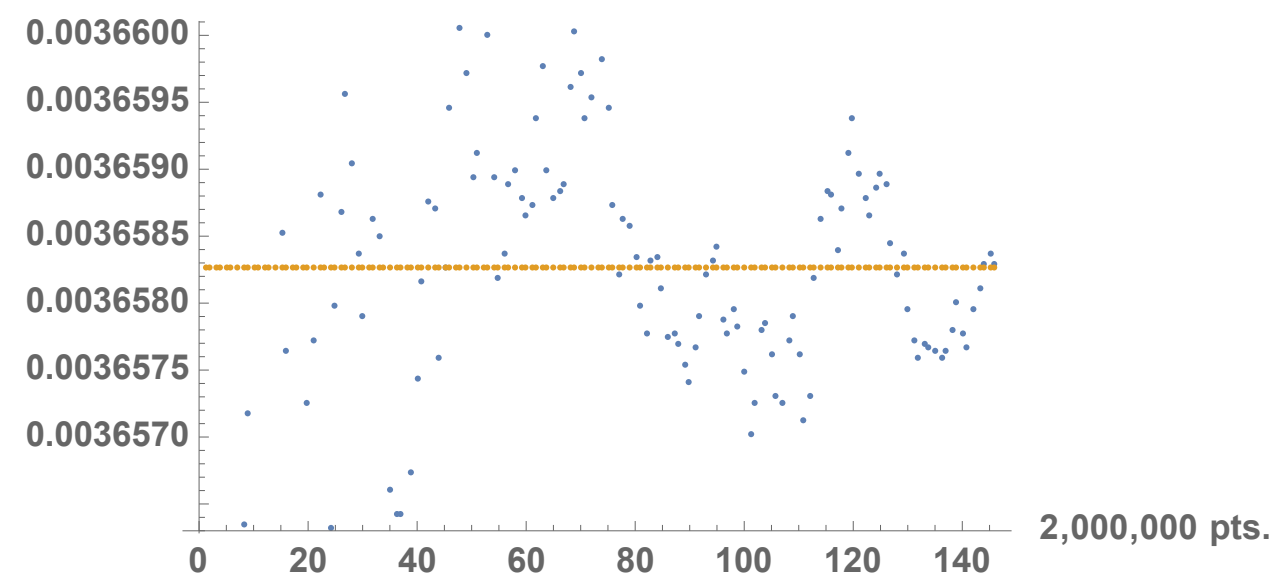

FIG. 11: Quasirandom estimation (with the Roberts parameter set to $\alpha_{0}=0$ ) of the Hilbert-Schmidt two-qubit absolute separability probability along with the predicted value A2.

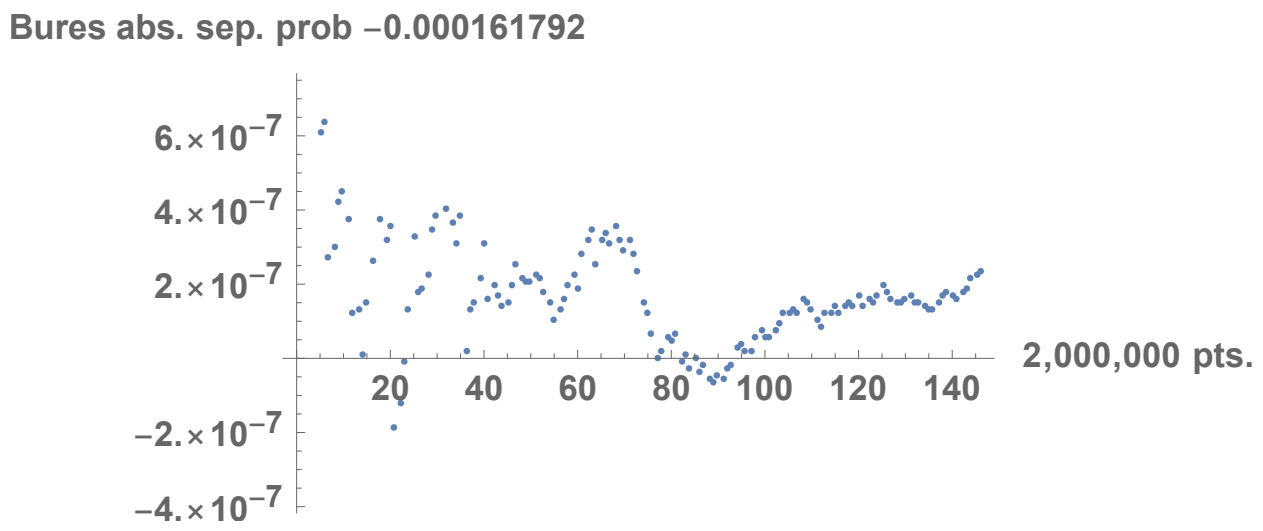

FIG. 12: Deviations about the previously tabulated value of 0.000161792 of a quasirandom estimation (with $\alpha_{0}=0$ ) of the Bures two-qubit absolute separability probability

Our last (presumably most precise) quasirandom estimates of the absolute separability probabilities with respect to the Kubo-Mori, Wigner-Yanase and identric measures are $5.31648 \times 10^{-6}, 0.0000343464$ and 0.000076423 , respectively.

Independent 4-dimensional, more conventional-type, numerical integrations gave $5.04898 \times$ $10^{-6}, 0.0000342309$ and 0.0000762634 for the Kubo-Mori, Wigner-Yanase and identric absolute separability probabilities.

For the $k=1$ case of induced measure $(k=0$ corresponding to the Hilbert-Schmidt instance), for which the two-qubit separabilty probability is $\frac{61}{143}=\frac{61}{11.13} \approx 0.426573$ [5], the absolute separability probability is $\approx 0.0232545$. For $k=2$, the corresponding pair of 
Kubo-Mori abs. sep. prob.

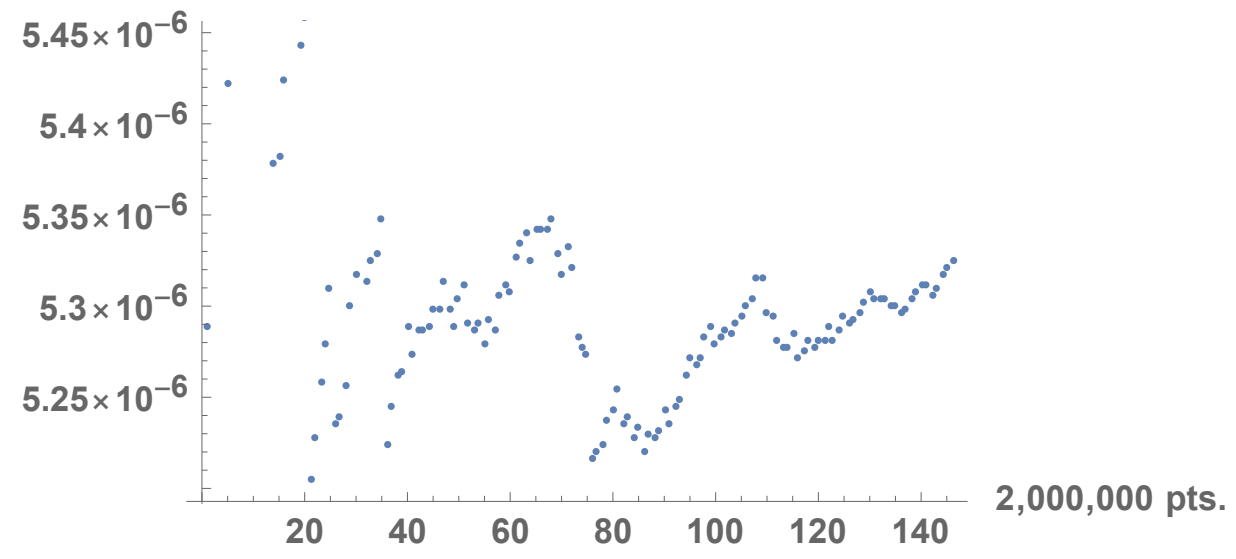

FIG. 13: Quasirandom estimation (with $\alpha_{0}=0$ ) of the Kubo-Mori two-qubit absolute separability probability

GKS/identric abs. sep. prob.

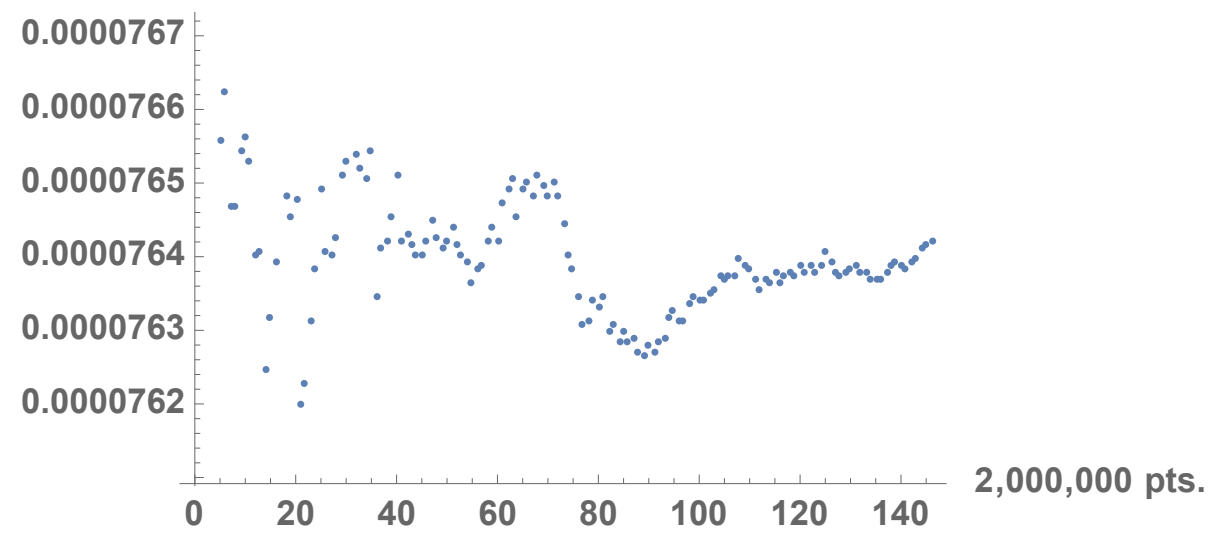

FIG. 14: Quasirandom estimation (with $\alpha_{0}=0$ ) of the GKS/identric two-qubit absolute separability probability

probabilities is $\frac{259}{442}$ and $\approx 0.071066971$. For $k=3,4$, the absolute separability probabilities increase substantially to approximately 0.1499309 and 0.252828 .

In Fig. 15 we plot the absolute separability probability as the induced measure parameter $k=K-4(N=4)$ increases from the Hilbert-Schmidt setting of $k=0$, at which the probability is given by A2). ("The natural, rotationally invariant measure on the set of all pure states of a $N \times K$ composite system, induces a unique measure in the space of $N \times N$ mixed states" [24]. The parameter $k$ is the difference $[k=K-N]$ between the dimensions $[K, N$,with $K \geq N]$ of the subsystems of the pure state bipartite system in which the density 
abs. sep. prob.

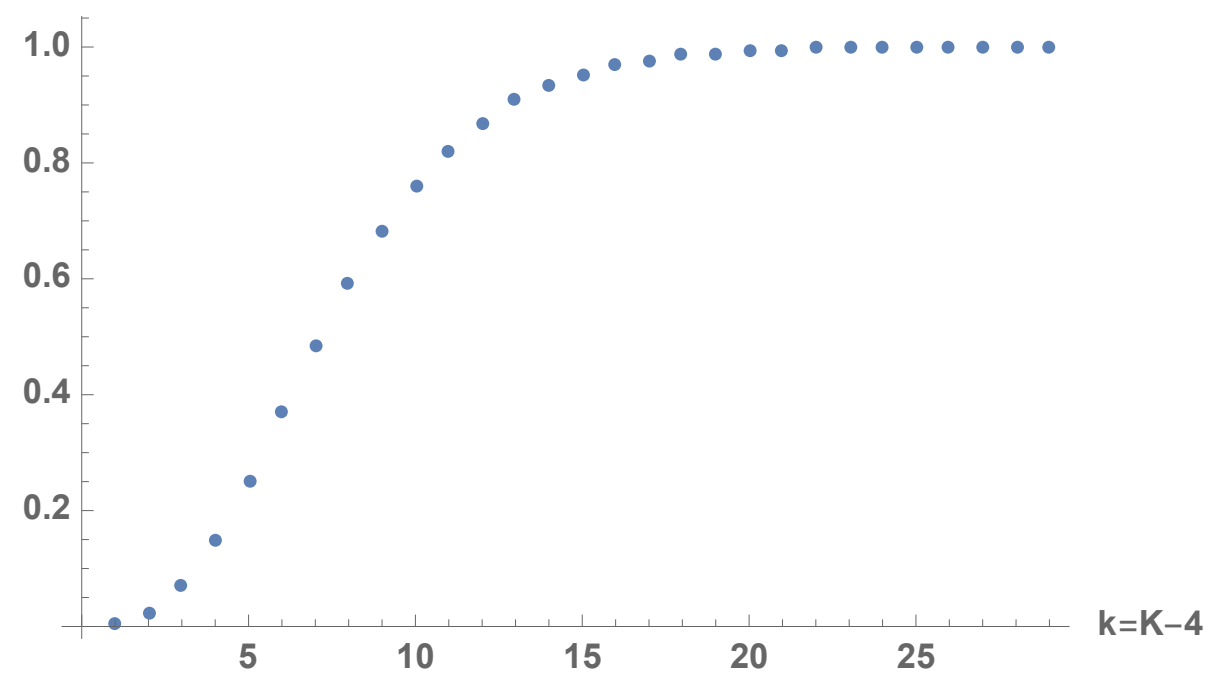

FIG. 15: Increase in the absolute separability probability as the induced measure parameter $k=K-4$ increases from the Hilbert-Schmidt value of $k=0$, at which the probability is given by A2

matrix is regarded as being embedded [24].)

\section{Variation with Bloch radius of qubit subsystems}

In Fig. 16 we show the Hilbert-Schmidt two-qubit absolute separability probabilitygiven by A2 -as a function of the Bloch radii of the reduced qubit subsystems. In the (total/absolute and non-absolute) Hilbert-Schmidt separability probability case-by results of Lovas-Andai and Milz-Strunz [3, 6]-the corresponding curve is flat at the value of $\frac{8}{33}$. (An effort to produce a corresponding plot in the qubit-qutrit case-where the eigenvalue condition $\lambda_{1}-\lambda_{5}-2 \sqrt{\lambda_{1} \lambda_{6}} \leq 0$ would be implemented-proved somewhat problematical as realizations, meeting this requirement-of absolutely separable states were very rare.) 


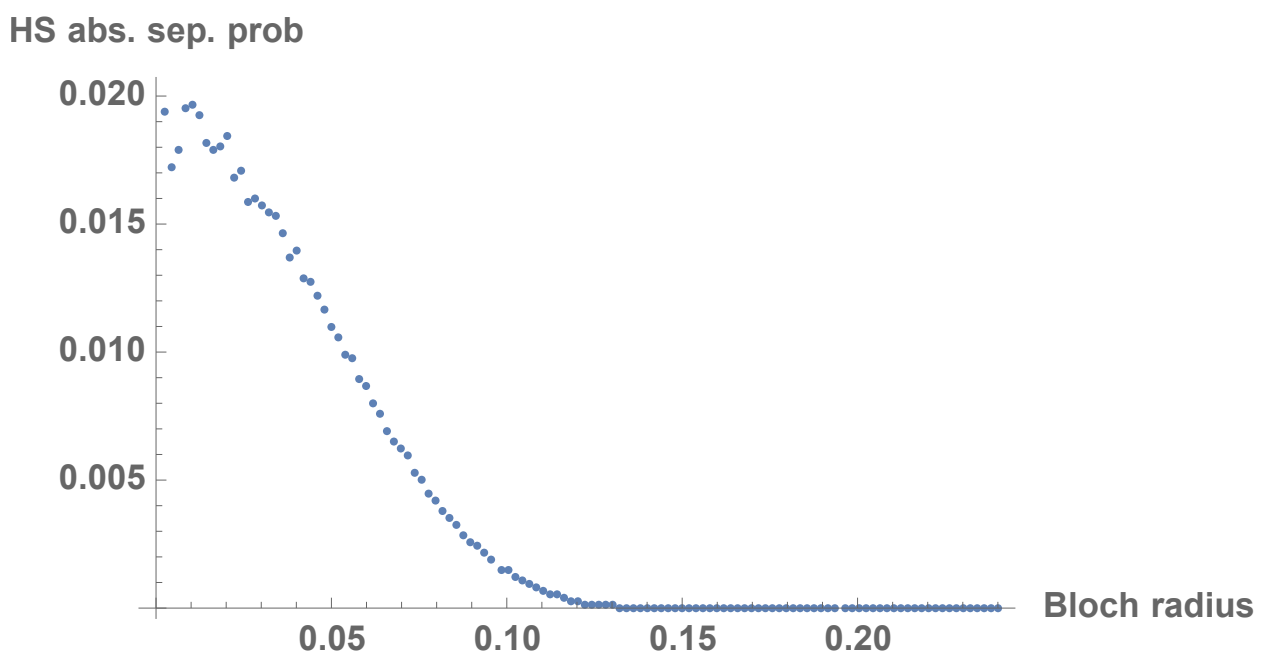

FIG. 16: Hilbert-Schmidt two-qubit absolute separability probability as a function of the Bloch radii of the reduced qubit subsystems. Without the absoluteness requirement, the curve is flat at $\frac{8}{33}$.

\section{Acknowledgments}

This research was supported by the National Science Foundation under Grant No. NSF PHY-1748958.

[1] P. B. Slater, Quantum Information Processing 17, 83 (2018).

[2] K. Życzkowski, P. Horodecki, A. Sanpera, and M. Lewenstein, Phys. Rev. A 58, 883 (1998).

[3] A. Lovas and A. Andai, Journal of Physics A: Mathematical and Theoretical 50, 295303 (2017).

[4] M. Ozawa, Phys. Lett. A 268, 158 (2000).

[5] P. B. Slater, arXiv preprint arXiv:1803.10680 (2018).

[6] S. Milz and W. T. Strunz, J. Phys. A 48, 035306 (2015).

[7] P. B. Slater, Quantum Information Processing 15, 3745 (2016).

[8] P. B. Slater, Journal of Geometry and Physics 59, 17 (2009).

[9] A. Andai, Journal of Physics A: Mathematical and General 39, 13641 (2006).

[10] D. Petz and C. Sudár, J. Math. Phys. 37, 2662 (1996).

[11] P. Gibilisco and T. Isola, Journal of Mathematical Physics 44, 3752 (2003).

[12] P. B. Slater, J. Phys. A 46, 445302 (2013).

[13] P. B. Slater, Quantum Information Processing 18, 312 (2019). 
[14] P. B. Slater, J. Geom. Phys. 53, 74 (2005).

[15] P. B. Slater, Phys. Rev. A 71, 052319 (2005).

[16] G. Ökten, MATHEMATICA in Educ. Res. 8, 52 (1999).

[17] P. B. Slater, J. Geom. Phys. 59, 17 (2009).

[18] T. Tilma, M. Byrd, and E. Sudarshan, Journal of Physics A: Mathematical and General 35, 10445 (2002).

[19] P. B. Slater, J. Geom. Phys. 58, 1101 (2008).

[20] P. B. Slater, J. Phys. A 32, 5261 (1999).

[21] P. B. Slater, Journal of Physics A: Mathematical and Theoretical 45, 455303 (2012), URL https://doi.org/10.1088\%2F 1751-8113\%2F45\%2F45\%2F455303.

[22] P. B. Slater, Quantum Information Processing 18, 121 (2019), ISSN 1573-1332, URL https: //doi.org/10.1007/s11128-019-2230-9.

[23] V. A. Osipov, H.-J. Sommers, and K. Życzkowski, J. Phys. A 43, 055302 (2010).

[24] K. Życzkowski and H.-J. Sommers, J. Phys. A 34, 7111 (2001).

[25] The unreasonable effectiveness of quasirandom sequences, URL http://extremelearning. com.au/unreasonable-effectiveness-of-quasirandom-sequences/.

[26] How can one generate an open ended sequence of low discrepancy points in 3d?, URL https://math.stackexchange.com/questions/2231391/ how-can-one-generate-an-open-ended-sequence-of-low-discrepancy-points-in-3d.

[27] M. Livio, The golden ratio: The story of phi, the world's most astonishing number (Broadway Books, 2008).

[28] Can i use compile to speed up inversecdf?, URL https://mathematica.stackexchange.com/ questions/181099/can-i-use-compile-to-speed-up-inversecdf.

[29] N. Tonchev, Journal of Mathematical Physics 57, 071903 (2016).

[30] C. Krattenthaler and P. B. Slater, IEEE Transactions on Information Theory 46, 801 (2000).

[31] R. Hildebrand, Physical Review A 76, 052325 (2007).

[32] M. Kuś and K. Życzkowski, Physical Review A 63, 032307 (2001).

[33] S. Arunachalam, N. Johnston, and V. Russo, arXiv preprint arXiv:1405.5853 (2014).

[34] A. Khvedelidze and I. Rogojin, Journal of Mathematical Sciences 209, 988 (2015). 